doi: $10.35366 / 100000$

\title{
Asociación de ferritina con deterioro ventilatorio y mortalidad debido a COVID-19 en terapia intensiva
}

Association of ferritin with ventilatory impairment and mortality due to COVID-19 in intensive care

Associação da ferritina com a deterioração ventilatória

e mortalidade devido ao COVID-19 na UTI

\section{Cesar Galicia García, ${ }^{\star}$ Gabriela Patricia Santana Hernández, ${ }^{*}$ Ángel Emmanuel Vega Sánchez*}

\section{RESUMEN}

Introducción: Se ha estudiado la asociación de ferritina sérica como parámetro de mal pronóstico y supervivencia en pacientes con COVID-19. Estudios afirman una supervivencia disminuida y mal pronóstico en pacientes con COVID y cifras de ferritina mayores de $1,000 \mathrm{ng} / \mathrm{mL}$. Esto implica que la hemoglobina está disminuyendo y el grupo hemo está aumentando, por lo tanto el cuerpo acumulará demasiados iones de hierro dañinos, lo que causará inflamación en el cuerpo y aumentará la proteína C-reactiva y la albúmina. Las células reaccionan al estrés debido a la inflamación, produciendo grandes cantidades de ferritina sérica para unir iones de hierro libres con el fin de reducir el daño.

Objetivo: El objetivo del estudio fue evaluar la ferritina sérica medida al ingreso como predictor de evolución en la $\mathrm{UCI}$, para lo cual se tomaron como desenlaces principales intubuación y muerte. Se realizó un cálculo de sensibilidad y especificidad y se estimó el área bajo la curva por medio de la curva ROC. Se evaluó la fuerza de asociación de la ferritina por medio del cálculo del OR crudo y ajustado por regresión logística univariada multivariada. Se evaluó la ferritina como marcador pronóstico de intubación y mortalidad en pacientes ingresados en terapia intensiva.

Material y métodos: Se realizó un estudio retrospectivo, transversal, en el cual se reclutaron 48 pacientes en la Terapia Intensiva del Hospital San Ángel Inn Universidad, cuyos datos clínicos y variables de interés se tomaron del expediente clínico. Se realizó una descripción de los datos con base en su distribución en medias con desviación estándar o medianas con rangos intercuartilares de las variables continuas, y de los datos categóricos en frecuencias con porcentaje.

Resultados: Para mortalidad se escogió de la curva ROC un valor de sensibilidad de $50 \%$, especificidad de 88.89 , LR + de 4.5 y LR - de 0.56 , con ello el área fue de ocho utilizando este valor de corte, la proporción de pacientes con mortalidad bajo este valor fue de 7.8 , el riesgo de mortalidad y sensibilidad fue de $72.73 \%$, especificidad de 73.08 , LR + de 2.7 y LR - de 0.37 , con ello el área fue de 7.2 utilizando este valor de corte, la proporción de pacientes intubados bajo este valor fue de 9.3, con ello se realizó una regresión logística univariada observando un OR crudo de 8 y ajustado de 7.8 para mortalidad, y un OR crudo de 7.23 y ajustado de 9.3 para intubación, y ajustado a confusores para edad con un OR de 1.0, con valor de $p$ de 0.080 , hipertensión con un OR de 3.2, valor de $\mathrm{p}$ de 0.10 y para obesidad con un OR de 1.89 , con valor de $\mathrm{p}$ de 0.041 .

Conclusión: En los pacientes ingresados al hospital San Ángel Inn Universidad con criterios de gravedad para terapia intensiva, la presencia de ferritina mayor de $1,100 \mathrm{ng} / \mathrm{mL}$ tiene una sensibilidad de $72.73 \%$, especificidad de $73.08 \%$, LR + de 2.70 y LR - de 0.37, este valor se asoció a intubación con un OR crudo de 7.2 y ajustado de 9.3 y la presencia de ferritina mayor de $2,507 \mathrm{ng} / \mathrm{mL}$ tiene una sensibilidad de $50 \%$, especificidad de $88.89 \%$, LR + de 4.5 y LR - de 0.56 , este valor se asoció a mortalidad con un OR crudo de 8 y ajustado de 7.8. Por tal motivo, un valor de corte de ferritina superior a 1,100 en pacientes ingresados a $\mathrm{UCl}$ debe ser considerado como un pronóstico para intubación durante el internamiento. Este marcador debe ser evaluado con mayor número de pacientes para comprobar su utilidad.

Palabras clave: Ferritina, mortalidad, intubación, supervivencia, pronóstico.

\section{* Hospital San Ángel Inn Universidad, CDMX.}

Recibido: 09/10/2020. Aceptado: 21/10/2020.

Citar como: Galicia GC, Santana HGP, Vega SÁE. Asociación de ferritina con deterioro ventilatorio y mortalidad debido a COVID-19 en terapia intensiva. Med Crit. 2021;35(3):121-129. https://dx.doi.org/10.35366/100000

www.medigraphic.com/medicinacritica

\section{ABSTRACT}

Introduction: The association of serum ferritin as a parameter of poor prognosis and survival has been studied in patients with COVID-19. Studies affirm a decreased survival and poor prognosis in patients with COVID and ferritin levels greater than $1,000 \mathrm{ng} / \mathrm{mL}$. This implies that the hemoglobin is decreasing, and the heme group is increasing, therefore the body will accumulate too many harmful iron ions, which will cause inflammation in the body and increase the C-reactive protein and albumin. Cells react to stress due to inflammation, producing large amounts of serum ferritin to bind free iron ions to reduce damage.

Objetive: The objective of the study was to evaluate serum ferritin measured at admission as a predictor of evolution in the ICU, for which intubation and death were taken as the main outcomes. A sensitivity and specificity calculation were performed and the area under the curve was calculated by means of the ROC curve. The strength of association of ferritin was evaluated by calculating the crude $O R$ and adjusted by multivariate univariate logistic regression. Ferritin was evaluated as a prognostic marker of intubation and mortality in patients admitted to intensive care.

Material and methods: A retrospective, cross-sectional study was carried out, in which 48 patients were recruited in the intensive care unit of the San Angel Inn University Hospital. Which were taken the clinical data and variables of interest from the clinical record. A description of the data was made based on its distribution in means with standard deviation or medians with interquartile ranges of the continuous variables and in frequencies with percentage of the categorical data. Results: For mortality it was chosen from the ROC curve with a sensitivity value of $50 \%$, specificity $88.89, L R+$ of 4.5 and $L R-0.56$, with it the area was 8 using this cut-off value, the proportion of patients with mortality below this value was 7.8 for mortality risk and sensitivity of $72.73 \%$, specificity 73.08 , $L R+$ of 2.7 and $L R-0.37$, with this the area was 7.2 using this The cut-off value of the proportion of patients intubated under this value was 9.3, with this a univariate logistic regression was performed, observing a crude OR of 8 and adjusted for mortality, and crude OR of 7.23 and adjusted for 9.3 for intubation and adjusted to confounders for age with OR 1.0, with $p$ value of 0.080 , hypertension with OR of $3.2, p$ value of 0.10 and for obesity with $O R$ of 1.89 , with $p$ value of 0.041 .

Conclusion: In patients admitted to the San Angel Inn University hospital with severity criteria for intensive therapy, the presence of ferritin greater than 1100 $\mathrm{ng} / \mathrm{ml}$ has a sensitivity of $72.73 \%$, specificity of $73.08 \%$ and $L R+$ of 2.70 and $L R$ - of 0.37 of this value was associated with intubation with crude $O R$ of 7.2 and adjusted of 9.3 and the presence of ferritin greater than $2507 \mathrm{ng} /$ $\mathrm{ml}$, it has a sensitivity of $50 \%$, specificity of $88.89 \%$ and $L R+$ of 4.5 and $L R-$ of 0.56 of This value was associated with mortality with a crude OR of 8 and adjusted for 7.8. For this reason. A ferritin cut-off value greater than 1100, in patients admitted to ICU. It should be considered as a prognosis for intubation during hospitalization. This marker should be evaluated with a greater number of patients to verify its usefulness.

Keywords: Ferritin, mortality, intubation, survival, prognosis.

\section{RESUMO}

Introdução: A associação da ferritina sérica como parâmetro de mau prognóstico e de sobrevida tem sido estudada em pacientes com COVID-19. Estudos afirmam uma sobrevida diminuída e prognóstico ruim em pacientes com COVID e níveis de ferritina maiores que $1,000 \mathrm{ng} / \mathrm{mL}$. Isso implica que a hemoglobina está diminuindo e o grupo heme está aumentando; portanto, o corpo acumulará muitos íons de ferro prejudiciais, o que causará inflamação no corpo e aumentará a proteína $C$ reativa e a albumina. As células reagem ao estresse devido à inflamação, produzindo grandes quantidades de ferritina sérica para ligar os íons de ferro livres e reduzir os danos.

Objetivo: O objetivo do estudo foi avaliar a ferritina sérica medida na admissão como preditor de evolução na UTI, tendo intubação e óbito como desfechos principais. Foi realizado cálculo de sensibilidade e especificidade e calculada a área sob a curva por meio da curva ROC. A força de associação da ferritina 
foi avaliada pelo cálculo do OR bruto e ajustada por regressão logística multivariada univariada. A ferritina foi avaliada como marcador prognóstico de intubação e mortalidade em pacientes internados em terapia intensiva.

Material e métodos: Foi realizado um estudo transversal retrospectivo, no qual 48 pacientes foram recrutados na unidade de terapia intensiva do Hospital San Angel Inn Universidad. Os dados clínicos e as variáveis de interesse foram obtidos do prontuário clínico. A descrição dos dados foi feita a partir de sua distribuição em médias com desvio padrão ou medianas com intervalos interquartis das variáveis contínuas e em frequências com porcentagem dos dados categóricos.

Resultados: Por mortalidade, sendo escolhida a partir da curva ROC com valor de sensibilidade de $50 \%$, especificidade de 88.89, $L R+$ de 4.5 e $L R-0.56$ com ela a área foi 8 usando este valor de corte, a proporção de pacientes com mortalidade abaixo desse valor foi de 7.8 para risco de mortalidade $e$ sensibilidade de $72.73 \%$, especificidade 73.08, $L R+$ de 2.7 e $L R-0.37$, com isso a área foi de 7.2 usando este o valor de corte da proporção de pacientes intubados sob este valor foi de 9.3, com isso foi realizada uma regressão logística univariada, observando um OR bruto de 8 e ajustado para mortalidade, e OR bruto de 7.23 e ajustado para 9.3 para intubação e ajustado para confundidores para idade com OR 1.0, com valor de $p$ de 0.080 , hipertensão com OR de 3.2, valor de $p$ de 0.10 e para obesidade com OR de 1.89, com valor de $p$ de 0.041 .

Conclusão: Em pacientes internados no Hospital San Ángel Inn Universidad com critérios de gravidade para Terapia Intensiva, a presença de ferritina maio que $1,100 \mathrm{ng} / \mathrm{mL}$, tem sensibilidade de $72.73 \%$, especificidade de $73.08 \%$ e LR + de 2.70 e $L R$ - de 0.37 deste valor foi associado à intubação com OR bruto de 7.2 e ajustado de 9.3 e à presença de ferritina maior que 2,507 ng/mL, tem sensibilidade de 50\%, especificidade de $88.89 \%$ e $L R+$ de 4.5 e $L R-$ de 0.56 desse valor foi associado à mortalidade com um OR bruto de 8 e ajustado para 7.8. Por tal motivo. Um valor de corte de ferritina superior a 1,100, em pacientes admitidos na UTI. Deve ser considerado como um prognóstico para intubação durante a hospitalização. Esse marcador deve ser avaliado com um número maior de pacientes para verificar sua utilidade.

Palavras-chave: Ferritina, mortalidade, intubação, sobrevivência, prognóstico.

\section{INTRODUCCIÓN}

A finales de 2019 se identificó un nuevo coronavirus como la causa de un grupo de casos de neumonía en Wuhan, China. Desde entonces se ha extendido rápidamente, lo que ha resultado en una pandemia. La Organización Mundial de la Salud designó el término de enfermedad COVID-19 (es decir, enfermedad por coronavirus 2019). El virus que causa COVID-19 se denomina coronavirus del síndrome respiratorio agudo severo (SARS-CoV-2). La mayor morbilidad y mortalidad por COVID-19 se debe en gran parte a la neumonitis viral aguda que evoluciona a síndrome de dificultad respiratoria aguda (SDRA). ${ }^{1,2}$

Los coronavirus humanos a través de su proteína espiga se unen a uno de los receptores, éstos pueden ser: la enzima convertidora de angiotensina, dipeptidil peptidasa 4, aminopeptidasa $\mathrm{N}$ y O-ácido acetilsalićlico, e ingresan a la célula a través de una vía endosómica y/o no endosómica; una vez ingresados, se liberan la nucleocápside y el ARN en el citoplasma, se sintetizan las enzimas que participan en la transcripción y replicación del virus, se producen copias ARN de sentido negativo, por medio del ARN subgenómico se producen las proteínas estructurales que posteriormente serán ensambladas y se libera el virión a través de exocitosis al espacio extracelular.

La ferritina es un mediador clave de la desregulación inmune, especialmente bajo hiperferritinemia extrema, a través de efectos inmunosupresores y proinflamatorios directos que contribuyen a la tormenta de citoquinas.
En un estudio con 20 pacientes con COVID-19 se descubrió que las personas con COVID-19 grave y muy grave exhibieron un aumento en el nivel de ferritina sérica, siendo la ferritina sérica en el grupo COVID-19 muy grave significativamente más alta que en el grupo COVID-19 grave (1006.16 ng/mL [RIC: 408.265-1988.25] vs. $291.13 \mathrm{ng} / \mathrm{mL}$ [RIC: 102.1-648.42], respectivamente). De acuerdo con esto, otro estudio reveló que en los pacientes que murieron por COVID-19, los niveles de ferritina fueron altos al ingreso hospitalario y durante toda la estadía en el hospital. Los valores medios de los niveles séricos de ferritina después del día 16 de hospitalización excedieron el límite superior de detección en estos pacientes, lo que sugiere que los niveles de ferritina aumentaron sin parar.

Además, Chen y colaboradores analizaron las características clínicas de 99 pacientes, de los cuales 63 tenían ferritina sérica muy por encima del rango normal. Se encontraron niveles elevados de ferritina también en autopsias de 12 pacientes cuya causa de muerte fue infección por SARS-CoV-2. Un análisis de la sangre periférica de 69 pacientes con COVID-19 grave reveló niveles elevados de ferritina en comparación con pacientes con enfermedad no grave. Por lo tanto, se concluyó que los niveles de ferritina sérica estaban estrechamente relacionados con la gravedad de COVID-19. ${ }^{3}$

El espectro clínico en los pacientes infectados por SARS-CoV-2 es amplio, incluye desde casos asintomáticos hasta neumonías graves e incluso la muerte. Las características clínicas de los primeros 425 casos confirmados tenían una mediana de edad de 59 años, con rangos de 15 a 89 años, $56 \%$ eran hombres, y en un inicio no se reportó ningún menor de 15 años. El periodo de incubación varía de dos a 21 días, con mayor frecuencia entre tres y siete días.

Aunque las tasas varían entre quienes están hospitalizados, hasta una cuarta parte necesita admisión en la unidad de cuidados intensivos (UCI), lo que representa aproximadamente de 5 a $8 \%$ de la población infectada total. 3,4

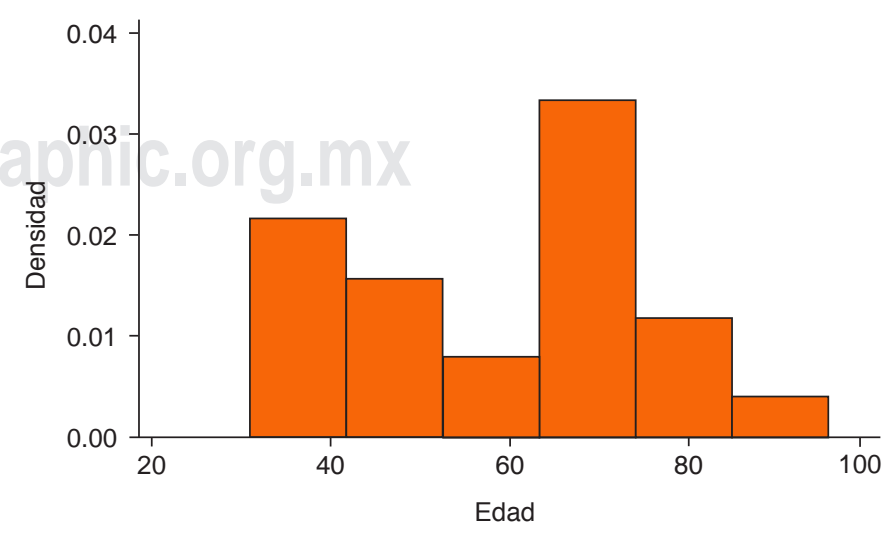

Figura 1: Comportamiento etario. 


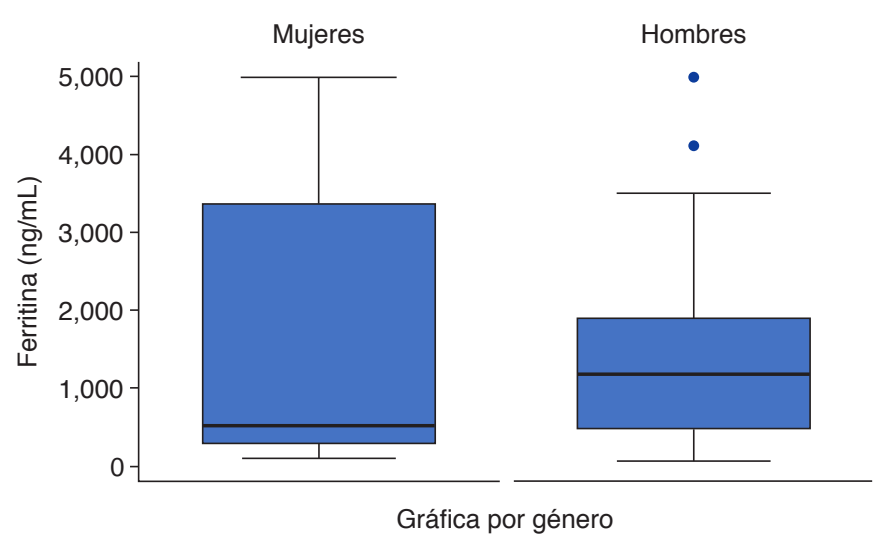

Figura 2: Comportamiento de ferritina sérica y sexo.

\section{Factores de riesgo de progresión}

La edad parece ser el principal factor de riesgo que predice la progresión a SDRA. Las comorbilidades, fiebre alta $\left(\geq 39^{\circ} \mathrm{C}\right)$, antecedentes de tabaquismo y características de laboratorio seleccionadas también predicen la progresión y la muerte por COVID-19. Es importante destacar que los adultos de cualquier edad pueden desarrollar enfermedades graves y experimentar resultados adversos, especialmente aquéllos con comorbilidades. Se proporcionan detalles adicionales sobre el riesgo de progresión de la enfermedad por separado..$^{5-8}$

Los hallazgos de laboratorio en pacientes críticos (por ejemplo, leucopenia, linfopenia, leucocitosis, dímero D elevado, lactato deshidrogenasa y ferritina, procalcitonina normal o baja) son inicialmente modestos y similares a aquéllos con enfermedad más leve, aunque el nivel de procalcitonina puede ser más elevado y la linfopenia más profunda en pacientes críti$\cos ^{3,8,9}$

Algunos pacientes con COVID-19 grave tienen evidencia de laboratorio de una respuesta inflamatoria exuberante, similar al síndrome de liberación de citocinas (SRC), con fiebres persistentes, marcadores inflamatorios elevados (p. ej. dímero $D$, ferritina, interleucina-6) y citocinas proinflamatorias elevadas; estas anormalidades de laboratorio se han asociado con mal pronóstico. ${ }^{10}$

Hay cada vez más pruebas de que pacientes críticos presentan características de hiperinflamación, que consisten en proteína $C$ reactiva en suero (PCR) elevada, procalcitonina (PCT), dímero D e hiperferritinemia. Estos hallazgos sugieren un papel posiblemente crucial de una tormenta de citoquinas en la fisiopatología de COVID-19.

Los marcadores inespecíficos de inflamación (p. ej. proteína $C$ reactiva $[P C R]$, ferritina) están universalmente elevados en el SARS-CoV-2 cuando se miden las citocinas inflamatorias ( $p$. ej. interferón gamma, inter- leucina [IL]-6, IL-10, IL-2R alfa soluble), también están elevadas. En general, el grado de elevación de las citocinas y los marcadores de inflamación se correlacionan con la gravedad del síndrome clínico. La elevación significativa de IL-6 es un hallazgo de apoyo para el diagnóstico de SARS-CoV-2. ${ }^{9-14}$

Los niveles elevados de PCR sérica, PCT, dímero $D$ y ferritina sérica se asociaron con un mal resultado compuesto aumentado que comprende mortalidad en pacientes con COVID-19, ARDS y la necesidad de atención en la unidad de cuidados intensivos.

Dentro de los marcadores descritos, la PCR, una proteína inflamatoria de fase aguda producida por el hígado, ha mostrado elevación en los procesos inflamatorios durante la enfermedad de COVID-19 grave y la necesidad de atención en la $\mathrm{UCl}$, pero no se ha relacionado con mortalidad. ${ }^{14}$

Otro marcador propuesto, un precursor peptídico de la hormona calcitonina, es la procalcitonina (PCT); sin embargo, en estudios anteriores se ha observado que las infecciones virales dificultan la producción de PCT debido a la producción de interferón, esto explica por qué las concentraciones séricas de PCT siguen siendo normales en casos no complicados de COVID-19, y los valores elevados pueden indicar coinfección bacteriana en casos severos, motivo por el cual se asocia una PCT sérica elevada con mortalidad en COVID-19 grave. Nuestro análisis SROC mostró el valor diagnóstico del suero PCT $0.5 \mathrm{ng} / \mathrm{mL}$ para un mal resultado compuesto en COVID-19 (88\% de sensibilidad, $68 \%$ de especificidad, LR +2.7 y LR-0.2)..$^{6,15}$

En un estudio encontramos también que un dímero D elevado se asoció con un aumento de mal resultado, especialmente la mortalidad y el COVID-19 grave. Este hallazgo respalda la hipótesis de que la infección por coronavirus 2 del síndrome respiratorio agudo severo (SARS-CoV-2) podría inducir la disfunción del sistema hemostático, lo que lleva a un estado hipercoagulable, una condición que comúnmente observamos en la sepsis. ${ }^{10,16}$

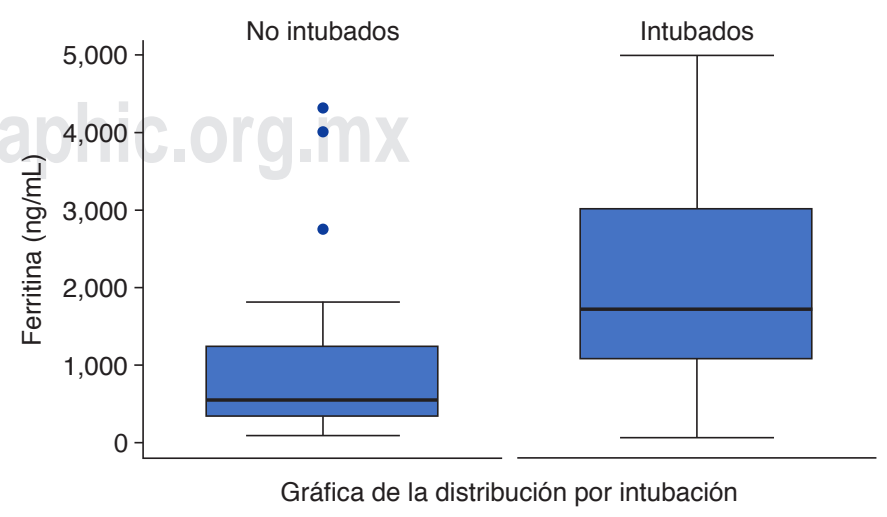

Figura 3: Comportamiento de ferritina e intubación. 


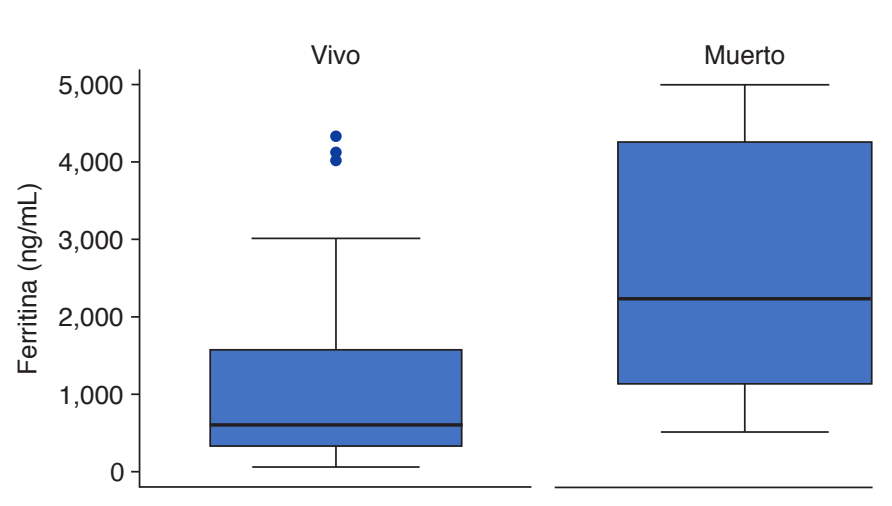

Gráfica de valores de ferritina por supervivencia

Figura 4: Comportamiento de ferritina sérica y mortalidad.

En este estudio se toma la ferritina sérica, ya que un valor más alto se asocia independientemente a SDRA, mortalidad y COVID-19 grave.

La ferritina $\mathrm{H}$ parece mostrar no sólo una función inmunomoduladora, sino también una actividad inflamatoria que culmina con la inducción de la expresión de diferentes mediadores inflamatorios, incluida IL-1 $\beta$.

SHLH es una condición de hiperinflamación caracterizada por una tormenta de citoquinas que causa falla fatal de múltiples órganos. Esta condición se desencadena con mayor frecuencia por infecciones virales, lo que podría conducir a una hipótesis de SARS-CoV-2 que induce este síndrome hiperinflamatorio.

Similitudes clínicas, de laboratorio y automáticas: COVID-19 vs. síndromes hiperferritinémicos donde se aprecia la elevación de ferritina que oscila de 300 a $5,000 \mathrm{ng} / \mathrm{mL}$, asimismo se aprecia hipercitoquinemia, fiebre en la mayoría de los casos, coagulación intravascular diseminada, síndrome de distrés respiratorio agudo y leucopenia en algunos casos como manifestaciones graves de COVID-19.

Además de una secreción activa durante la reacción inflamatoria, un componente principal de la ferritina sérica deriva de la muerte celular y, en particular, de la muerte de las células hepáticas. Una vez liberada, la ferritina pierde parte del contenido interno de hierro, dando lugar a niveles séricos extremadamente altos de «hierro libre». Parece que el exceso de circulación de «hierro libre» detectable durante la fase severa en condiciones inflamatorias puede deteriorar la reacción inflamatoria con la capacidad particular de inducir un marcado estado procoagulante. ${ }^{17}$

\section{MATERIAL Y MÉTODOS}

\section{Planteamiento del problema}

La enfermedad por infección por virus SARS-CoV-2 se le ha relacionado a un fuerte impacto de salud mundial, además de gran mortalidad asociada a pacientes con comorbilidades, por lo que sería de utilidad contar con un marcador de gravedad al ingreso en estos pacientes. Contamos con varios marcadores de inflamación, siendo la ferritina uno de los más importantes, por lo que nos planteamos la siguiente pregunta de investigación.

¿Cuál es la asociación de las cifras de ferritina sérica de ingreso con la mortalidad y el riesgo de intubación de los pacientes ingresados en unidad de cuidados intensivos?

\section{Justificación}

En el contexto de una enfermedad nueva con una mortalidad elevada de los pacientes ingresados a la $\mathrm{UCI}$ es imprescindible contar con predictores tempranos de mal pronóstico, por tal motivo la exploración de marcadores es relevante, y basados en la fisiopatología de la enfermedad la ferritina es un valor sugerido con la capacidad de predicción en SARS-CoV-2.

\section{Hipótesis alterna}

¿Existe un valor de corte para la ferritina sérica tomada al ingreso en pacientes ingresados a la $\mathrm{UCl}$ que tenga utilidad en la predicción para intubación y muerte en los pacientes de $\mathrm{UCl}$ ? Hipótesis nula. ¿Existe un valor de corte para la ferritina sérica tomada al ingreso en pacientes ingresados a la $\mathrm{UCI}$ que tenga utilidad en la predicción para intubación y muerte en dichos pacientes?

\section{Metodología}

a. Población y tamaño de la muestra: se incluyeron pacientes con diagnóstico de enfermedad por COVID-19 ingresados en la Terapia Intensiva del Hospital San Ángel Inn Universidad, siendo un total

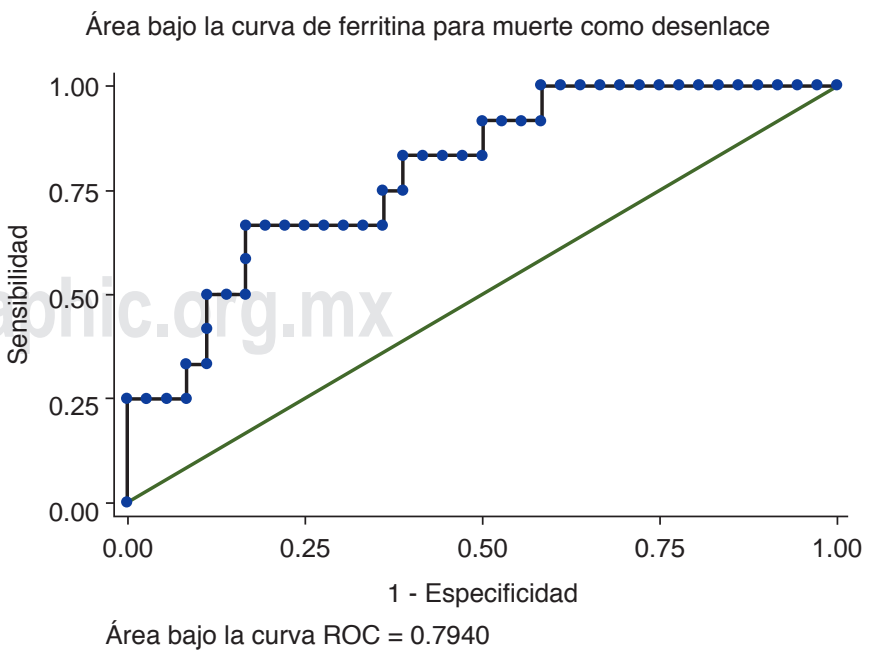

Figura 5: Curva ROC ferritina y mortalidad.

ROC $=$ Receiver Operating Characteristic. 
Área bajo la curva de ferritina para intubación

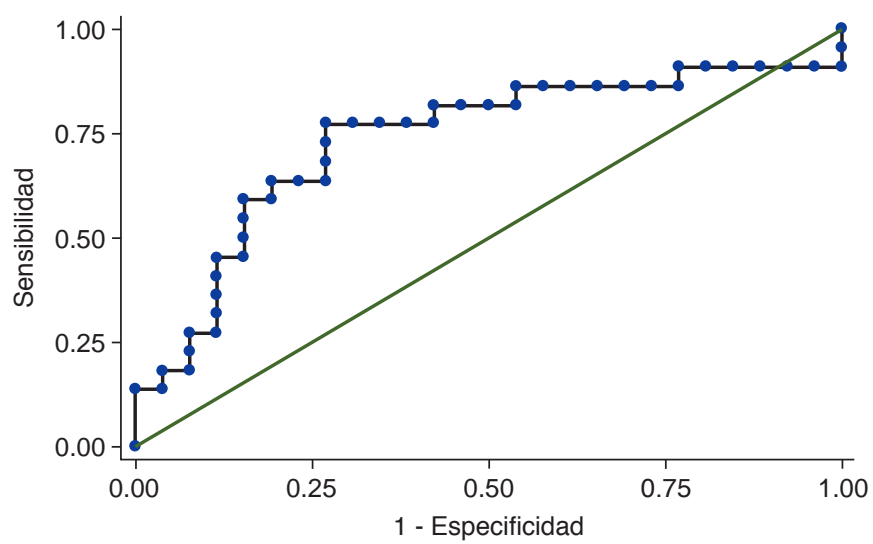

Área bajo la curva $\mathrm{ROC}=0.7343$

Figura 6: Curva ROC ferritina e intubación. $\mathrm{ROC}=$ Receiver Operating Characteristic.

de 48 pacientes. Se realizó reclutamiento consecutivo dentro del periodo de marzo 15 de 2020 a mayo 31 de 2020.

b. Criterios de inclusión: pacientes mayores de 18 años con diagnóstico de neumonía por COVID-19 que ingresen a terapia intensiva de esta unidad hospitalaria, los cuales cuenten con PCR positiva para SARS-CoV-2 y marcadores inflamatorios al ingreso. c. Criterios de exclusión: todos los pacientes menores de 18 años y aquéllos sin diagnóstico serológico confirmado de neumonía por COVID-19 o que no cuenten con marcadores al ingreso.

\section{d. Definición de variables: (Tabla 1)}

e. Procedimiento: se identificarán todos los pacientes mayores de 18 años con diagnóstico clínico serológico de COVID-19 que ingresen a esta unidad hospitalaria. Se procesará batería de laboratorios, incluyendo niveles de ferritina sérica y otros marcadores inflamatorios al ingreso a terapia intensiva.

Se determinará la distribución de normalidad en la muestra y las pruebas estadísticas de asociación entre variables, eliminando factores de confusión y se determinará el OR.

f. Análisis estadístico: estudio retrospectivo, transversal. Se reclutaron 48 pacientes en la Terapia Intensiva del Hospital San Ángel Inn Universidad cuyos datos clínicos y variables de interés se tomaron del expediente clínico. Se realizó una descripción de los datos con base en su distribución en medias con desviación estándar o medianas con rangos intercuartilares de las variables continuas, y de los datos categóricos en frecuencias con porcentaje. El objetivo del estudio fue evaluar la ferritina sérica medida al ingreso como predictor de evolución en la $\mathrm{UCI}$, para

Tabla 1: Definición de variables.

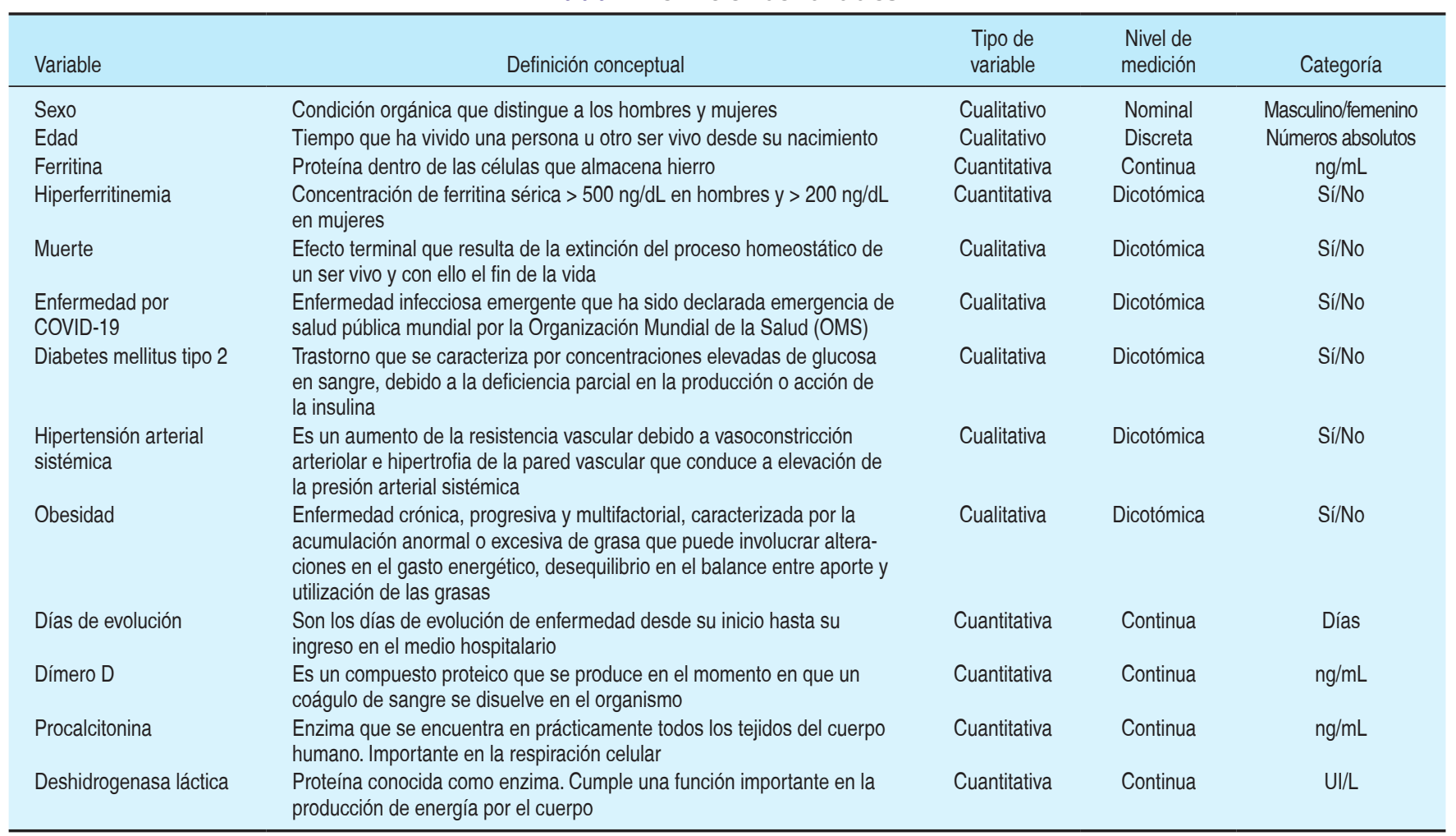


Tabla 2: Marcadores inflamatorios.

\begin{tabular}{lcrrr}
\hline & & & RIC & \\
\cline { 3 - 5 } & & $25 \%$ & $50 \%$ & $75 \%$ \\
\hline Ferritina & $+1,498.09 \pm 1,443.38$ & 418.00 & $1,044.99$ & 2,113 \\
Protéńn C reactiva & $+26.39 \pm 69.75$ & 5.15 & 12.15 & 25.45 \\
Dímero D & $+1,819.25 \pm 1,762.25$ & 425.00 & $1,008.50$ & $3,439.50$ \\
Deshidrogenasa láctica & $+835 \pm 2459.53$ & 281.50 & 426.00 & 602.00 \\
Procalcitonina & $+1.55 \pm 2.75$ & 0.2 & 0.26 & 1.57 \\
\hline
\end{tabular}

lo cual se tomaron como desenlaces principales intubuación y muerte. Se realizó un cálculo de sensibilidad y especificidad y se estimó el área bajo la curva por medio de la curva ROC. Se evaluó la fuerza de asociación de la ferritina por medio del cálculo del OR crudo y ajustado por regresión logística univariada multivariada.

1. Aspectos éticos y de bioseguridad:

a. Consentimiento informado.

b. Aprobación por el Comité de Ética del Hospital San Ángel Inn Universidad.

c. Norma Oficial Mexicana NOM-012-SSA3-2012 que establece los criterios para la ejecución de proyectos de investigación para la salud en seres humanos. Títulos: $6^{\circ}, 7^{\circ}, 8^{\circ}, 10^{\circ}, 11^{\circ}, 12^{\circ}, 13^{\circ} \mathrm{y}$ $14^{\circ}$.

d. Declaración de Helsinki de la AMM-Principios éticos para las investigaciones médicas en seres humanos, $64^{\mathrm{a}}$ Asamblea General, Fortaleza, Brasil, octubre 2013.

e. Reglamento de la Ley General de Salud en Materia de Investigación para la Salud, Título Segundo, Capítulo I, Artículo 17.

\section{Relevancia y expectativas:}

Contar con un marcador de gravedad al ingreso y aportar mayor vigilancia a estos pacientes con el objetivo de disminuir la mortalidad y costos hospitalarios.

\section{RESULTADOS}

Se reclutaron 48 pacientes con una media de edad de 58.45 años, de los cuales 36 fueron hombres (75\%) y 12 mujeres (25\%) (Figuras 1 y 2). Dentro de sus comorbilidades incluían diabetes mellitus 2 $(60.42 \%)$, hipertensión arterial sistémica $(60.42 \%)$ y obesidad $(70.43 \%)$. De estos pacientes 22 requirieron intubación durante su estancia, de los cuales $55.56 \%$ correspondió a hombres y $16.6 \%$ a mujeres. A su ingreso se analizaron los días de evolución con una media de $10 \%$ y una desviación estándar de 2.54 , presentando una mortalidad de $25 \%$ tanto en hombres como en mujeres.
Tabla 3: Pruebas estadísticas.

\begin{tabular}{|c|c|c|c|c|c|c|c|}
\hline & \multicolumn{3}{|c|}{ Hombre \% } & \multicolumn{3}{|c|}{ Mujer \% } & $p$ \\
\hline Diabetes & \multicolumn{3}{|c|}{63.89} & \multicolumn{3}{|c|}{50.00} & \\
\hline Hipertensión & \multicolumn{3}{|c|}{63.89} & \multicolumn{3}{|c|}{50.00} & \\
\hline Obesidad & \multicolumn{3}{|c|}{72.22} & \multicolumn{3}{|c|}{66.67} & \\
\hline Intubados & \multicolumn{3}{|c|}{55.56} & \multicolumn{3}{|c|}{16.67} & \\
\hline Fallecidos & \multicolumn{3}{|c|}{25.00} & \multicolumn{3}{|c|}{25.00} & \\
\hline Días de evolución & & & & & & & 0.51 \\
\hline$\%$ & 25 & 50 & 75 & 25 & 50 & 75 & \\
\hline Media & 8 & 9 & 11.5 & 8.5 & 10 & 12 & \\
\hline Desviación estándar & & 2.68 & & & 2.10 & & \\
\hline Ferritina & & & & & & & 0.46 \\
\hline$\%$ & 25 & 50 & 75 & 25 & 50 & 75 & \\
\hline Media & 480 & $1,170.5$ & $1,890.3$ & 288.5 & 520 & 3,370 & \\
\hline Desviación estándar & & $1,302.03$ & & & $1,869.8$ & & \\
\hline Intubación/ferritina & & & & & & & 0.0056 \\
\hline$\%$ & 25 & 50 & 75 & 25 & 50 & 75 & \\
\hline Media & $1,075.8$ & $1,725.5$ & $3,010.4$ & 342 & 551.5 & 1,241 & \\
\hline Desviación estándar & & $1,575.89$ & & & $1,110.5$ & & \\
\hline Ferritina/mortalidad & & & & & & & 0.0025 \\
\hline$\%$ & 25 & 50 & 75 & 25 & 50 & 75 & \\
\hline Media & $1,137.9$ & 2,236 & 4,250 & 339.2 & 606.5 & $1,570.5$ & \\
\hline Desviación estándar & & $1,699.19$ & & & $1,161.7$ & & \\
\hline
\end{tabular}

Dentro de las características de los marcadores de inflamación se obtuvieron los datos de las Tablas 2 y 3.

Se evaluó la ferritina como marcador pronóstico de intubación y mortalidad en pacientes ingresados en terapia intensiva (Figuras 3 a 6), para mortalidad se escogió de la curva ROC un valor de sensibilidad de $50 \%$, especificidad $88.89, \mathrm{LR}+$ de 4.5 y LR -0.56 , con ello el área fue de 8 utilizando este valor de corte, la proporción de pacientes con mortalidad bajo este valor fue de 7.8, el riesgo de mortalidad y sensibilidad fue de $72.73 \%$, especificidad 73.08 , LR + de 2.7 y LR -0.37 , con ello el área fue de 7.2 utilizando este valor de corte, la proporción de pacientes intubados bajo este valor fue de 9.3, con ello se realizó una regresión logística univariada observando un OR crudo de 8 y ajustado de 7.8 para mortalidad, y un OR crudo de 7.23 y ajustado de 9.3 para intubación, y ajustado a confusores para edad con un OR de 1.0, con valor de $p$ de 0.080 , hipertensión con un OR de 3.2, valor de $p$ de 0.10 y obesidad con un OR de 1.89 , con valor de $p$ de 0.041 y su correspondiente valor predictivo positivo (VPP) (Tabla 4). 
Tabla 4: Factores pronósticos para intubación con ferritina $>1,100 \mathrm{ng} / \mathrm{mL}$ y mortalidad con ferritina > 2,507 ng/mL al ingreso.

\begin{tabular}{lccccc} 
Nombre & ORC & IC 95\% & VPP & ORa & IC 95\% \\
\hline Muerte & 8.00 & $1.34-42.9$ & 0.0041 & 7.8 & $1.18-51.1$ \\
Intubación & 7.23 & $1.72-31.7$ & 0.0016 & 9.3 & $1.48-58.6$ \\
Edad & 1.00 & $0.99-1.10$ & 0.0800 & & 0.032 \\
Hipertensión & 3.20 & $0.77-13.3$ & 0.1000 & & \\
Obesidad & 1.89 & $0.40-8.73$ & 0.0410 & & \\
\hline
\end{tabular}

ORC = Odds Ratio crudo, IC 95\% = intervalo de confianza del 95\%, ORa = Odds Ratio ajustado, VPP = valor predictivo positivo.

\section{DISCUSIÓN}

Para realizar un análisis comparativo de las variables continuas entre grupos se llevó a cabo un análisis de estadística descriptiva, donde se obtuvieron los valores de la media por mortalidad por ferritina. Se realizó una descripción de los datos con base en su distribución en medias con desviación estándar o medianas con rangos intercuartilares de las variables continuas, y de los datos categóricos en frecuencias con porcentaje. De manera general, se observan diferencias entre grupos, por ejemplo, los valores de ferritina son en promedio mucho mayores en los pacientes que presentaron mortalidad y los pacientes que requirieron ventilación mecánica invasiva. De manera global, se observó una distribución casi normal en las variables en relación a la mortalidad. Se realizó un cálculo de sensibilidad y especificidad y se estimó el área bajo la curva por medio de la curva ROC. Se evaluó la fuerza de asociación de la ferritina por medio del cálculo del OR crudo y ajustado por regresión logística univariada multivariada, en la cual se obtuvieron los siguientes resultados: se reclutaron 48 pacientes con una media de edad de 58.45 años, de los cuales 36 fueron hombres (75\%) y 12 mujeres (25\%). Dentro de sus comorbilidades incluían diabetes mellitus $2(60.42 \%)$, hipertensión arterial sistémica $(60.42 \%)$ y obesidad (70.43\%). De estos pacientes 22 requirieron intubación durante su estancia, de los cuales $55.56 \%$ correspondió a hombres y $16.6 \%$ a mujeres. A su ingreso se analizaron los días de evolución con una media de $10 \%$ y una desviación estándar de 2.54, presentando una mortalidad de $25 \%$ tanto en hombres como en mujeres. Asimismo, se realizó la evaluación de la ferritina como marcador pronóstico de intubación y mortalidad en pacientes ingresados en terapia intensiva, para mortalidad se escogió de la curva ROC un valor de sensibilidad de $50 \%$, especificidad de 88.89 , LR + de 4.5 y LR - de 0.56 , con ello el área fue de 8 utilizando este valor de corte, la proporción de pacientes con mortalidad bajo este valor fue de 7.8 , el riesgo de mortalidad y sensibilidad fue de $72.73 \%$, especificidad de 73.08 , LR + de 2.7 y LR - de 0.37, con ello el área fue de 7.2 utilizando este valor de corte, la proporción de pacientes intubados bajo este valor fue de 9.3, con ello se realizó una regresión logística univariada observando un OR crudo de 8 y ajustado de 7.8 para mortalidad, y un OR crudo de 7.23 y ajustado de 9.3 para intubación, y ajustado a confusores para edad con un OR de 1.0, con valor de $\mathrm{p}$ de 0.080 , hipertensión con un OR de 3.2, valor de p de 0.10 y obesidad con un OR de 1.89, con valor de $p$ de 0.041 .

Se consideró a la ferritina como un marcador pronóstico de intubación y mortalidad en pacientes ingresados en terapia intensiva, lo cual traduce un buen trabajo de investigación.

\section{CONCLUSIÓN}

En los pacientes ingresados al hospital San Ángel Inn Universidad con criterios de gravedad para terapia intensiva, la presencia de ferritina mayor de 1,100 ng/ $\mathrm{mL}$ tiene una sensibilidad de $72.73 \%$, especificidad de $73.08 \%$, LR + de 2.70 y LR - de 0.37 , este valor se asoció a intubación con un OR crudo de 7.2 y ajustado de 9.3, y la presencia de ferritina mayor de 2,507 $\mathrm{ng} / \mathrm{mL}$ tiene una sensibilidad de $50 \%$, especificidad de $88.89 \%$, LR + de 4.5 y LR - de 0.56 , este valor se asoció a mortalidad con un OR crudo de 8 y ajustado de 7.8. Por tal motivo, los valores de la ferritina sérica tomados al ingreso parecen ser un fuerte marcador por arriba de 1,100 ng/mL para predecir intubación en pacientes ingresados a la UCl y con más fuerza en hombres. Con niveles por arriba de 2,500 ng/mL tiene gran capacidad para predecir la muerte. Sin embargo, estos resultados deben ser explorados en cortes más extensas para mejorar el ajuste a confusores e interacciones de los resultados.

\section{REFERENCIAS}

1. Ictvonline.org/ taxonomy, International Committee on taxonomy of viruses (ICTV). [Internet]. [Access 5 February of 2020] Available in: https://talk.ictvonline.org/ taxonomy/.

2. Su S, Wong G, Shi W, Liu J, Lai ACK, Zhou J, et al. Epidemiology, genetic recombination, and pathogenesis of coronaviruses. Trends Microbiol. 2016;24(6):490-502. doi: 10.1016/j.tim.2016.03.003.

3. Zhonghua Jie He He Hu Xi Za Zhi. Respiratory care committee of Chinese Thoracic Society. Expert consensus 
on preventing nosocomial transmission during respiratory care for critically ill patients infected by 2019 novel coronavirus pneumonia. Zhonghua Jie He He Hu Xi Za Zhi. 2020;43(4):288-296

4. Wang D, Hu B, Hu C, Zhu F, Liu X, Zhang J, et al. Clinical characteristics of 138 hospitalized patients with 2019 novel coronavirus-infected pneumonia in Wuhan, China. JAMA. 2020;323(11):1061-1069. doi:10.1001/jama.2020.1585.

5. Rosário C, Zandman-Goddard G, Meyron-Holtz EG, D'Cruz DP, Shoenfeld $Y$. The hyperferritinemic syndrome: macrophage activation syndrome, Still's disease, septic shock and catastrophic antiphospholipid syndrome. BMC Med. 2013;11:185.

6. Shoenfeld Y. Corona (COVID-19) time musings: our involvement in COVID-19 pa- thogenesis, diagnosis, treatment and vaccine planning. Autoimmun Rev. 2020;19(6):102538.

7. Siddiqi HK, Mehra MR. COVID-19 illness in native and immunosuppressed states: a clinical-therapeutic staging proposal. J Hearth Lung Transpl. 2020. Available in: https://doi. org/10.1016/j.healun.2020.03.012.

8. Alhazzani W, Hylander MM, Arabi YM, Loeb M, Ng GM, Fan E, et al. Surviving sepsis campaign: guidelines on the management of critically ill adults with coronavirus disease 2019 (COVID-19). Crit Care Med. 2020;28:1-34.

9. Conti P, Ronconi G, Caraffa A, Gallenga CE, Ross R, Frydas I. Induction of pro-inflammatory cytokines (IL-1 and IL-6) and lung inflammation by Coronavirus-19 (COVI-19 or SARS-CoV-2): anti-inflammatory strategies. J Biol Regul Homeost Agents. 2020;34(2):327-331.

10. Moroz C, Grunspan A, Zahalka MA, Traub L, Kodman Y, Yaniv I. Treatment of human bone marrow with recombinant placenta immunoregulator ferritin results in myelopoiesis and T-cell suppression through modulation of the cytokine-chemokine networks. Exp Hematol. 2006;34:159-166.

11. Zhou F, Yu T, Du R, Fan G, Liu Y, Liu Z. Clinical course and risk factors for mortality of adult inpatients with COVID-19 in Wuhan, China: a retrospective cohort study. Lancet. 2020;395(10229):1054-1062.

12. Ruddell RG, Hoang-Le D, Barwood JM, Rutherford PS, Piva TJ, Watters DJ, et al. Ferritin functions as a proinflammatory cytokine via iron-independent protein kinase C zeta/nuclear factor kappaB-regulated signaling in rat hepatic stellate cells. Hepatology. 2009;49:887-900.

13. Carter SJ, Tattersall RS, Ramanan AV. Macrophage activation syndrome in adults: recent advances in pathophysiology, diagnosis and treatment. Rheumatology (Oxford). 2019;58(1):5-17.

14. Inciardi RM, Lupi L, Zaccone G, Italia L, Raffo M, Tomasoni D. Cardiac involvement in a patient with coronavirus disease 2019 (COVID-19). JAMA Cardiol. 2020;5(7):819-824. Available in: https://doi.org/10.1001/jamacardio.2020.1096.

15. Shen $K$, Yang $Y$, Wang $T$, Zhao $D$, Jiang $Y$, Jin $R$, et al. Diagnosis, treatment, and prevention of 2019 novel coronavirus infection in children: experts' consensus statement. World J Pediatr. 2020;16(3):223-231. doi: 10.1007/ s12519-020-00343-7.

16. Wigginton JM. Reversal of ferritin-mediated immunosuppression by levamisole: a rationale for its application to management of the acquired immune deficiency syndrome (AIDS). Med Hypotheses. 1995;44:85-88

17. Mehta P, McAuley DF, Brown M, Sanchez E, Tattersall RS. Manson JJ. HLH across speciality collaboration, UK. COVID-19: consider cytokine storm syndromes and immunosuppression. Lancet. 2020;395(10229):1033-1034.

\section{LECTURAS RECOMENDADAS}

- Song Z, Xu Y, Bao L, Zhang L, Yu P, Qu Y, et al. From SARS to MERS, thrusting coronaviruses into the spotlight. Viruses. 2019;11(1):59. doi: 10.3390/v11010059.

- Zumla A, Chan JFW, Azhar El, Hui DSC, Yuen KY. Coronaviruses drug discovery and therapeutic options. Nat Rev Drug Discov. 2016;15(5):327-347. doi: 10.1038/nrd.2015.37.
- World Health Organization. Director-General's remarks at the media briefing on 2019-nCoV on 11 February 2020. (Accessed on February 12, 2020) https://www.who.int/dg/speeches/detail/ who-director-general-s-remarks-at-the-media-briefing-on-2019ncov-on-11-february-2020.

- www.cdc.gov.Centers for Disease Control and Prevention. 2019 Novel coronavirus, Wuhan, China. Information for Healthcare Professionals. [Accessed on February 14, 2020] Available in: https://www.cdc.gov/coronavirus/2019-nCoV/hcp/index.html.

- covid19treatmentguidelines.nih.gov, NIH COVID-19 Treatment Guidelines. [Accessed on April 22, 2020] Available in: https:// covid19treatmentguidelines.nih.gov/overview/management-ofcovid-19/.

- Wang L, Shi Y, Xiao T, Fu J, Feng X, Mu D, et al. Chinese expert consensus on the perinatal and neonatal management for the prevention and control of the 2019 novel coronavirus infection (First edition). Ann Trans/ Med. 2020;8(3):47. Available in: http:// dx.doi.org/10.21037/atm.2020.02.20.

- Chen N, Zhou M, Dong X, Qu J, Gong F, Han Y, et al. Epidemiological and clinical characteristics of 99 cases of 2019 novel coronavirus pneumonia in Wuhan, China: a descriptive study. Lancet. 2020;395(10223):507-513. doi: 10.1016/S01406736(20)30211-7.

- Yang X, Yu Y, Xu J, Shu H, Xia J, Liu H, et al. Clinical course and outcomes of critically ill patients with SARS-CoV-2 pneumonia in Wuhan, China: a single-centered, retrospective, observational study. Lancet Respir Med. 2020;8(5):475-481.

- Fuk-Woo J, Yuan S, Kok KH, Kai-Wang T, Chu H, Yang J, et al. A familial cluster of pneumonia associated with the 2019 novel coronavirus indicating person-to-person transmission: a study of a family cluster. Lancet. 2020;395(10223):514-523. doi: 10.1016/ S0140-6736(20)30154-9.

- Gabay C, Fautrel B, Rech J, Spertini F, Feist E, Kotter I, et al. Open-label, multicentre, dose-escalating phase II clinical trial on the safety and efficacy of tadekinig alfa (IL-18BP) in adult-onset Still's disease. Ann Rheum Dis. 2018;77(6):840-847.

- Zhang C, Wu Z, Li JW, Zhao H, Wang GQ. The cytokine release syndrome (CRS) of severe COVID-19 and Interleukin-6 receptor (IL-6R) antagonist tocilizumab may be the key to reduce the mortality. Int J Antimicrob Agents. 2020;55(5):105954.

- Nicastri E, Petrosillo N, Bartoli TA, Lepore L, Mondi A, Palmieri $F$, et al. National Institute for the Infectious Diseases "L. Spallanzani", IRCCS. recommendations for COVID-19 clinical management. Infect Dis Rep. 2020;12(1):8543.

- Zhang W, Zhao Y, Zhang F, Wang Q, Li T, Liu Z, et al. The use of anti-inflammatory drugs in the treatment of people with severe coronavirus disease 2019 (COVID-19): the experience of clinical immunologists from China. Clin Immunol. 2020;214:108393. Available in: https:// doi.org/10.1016/j.clim.2020.108393.

- Cohen LA, Gutierrez L, Weiss A, Leichtmann-Bardoogo Y, Zhang $\mathrm{DL}$, Crooks DR, et al. Serum ferritin is derived primarily from macrophages through a non-classical secretory pathway. Blood. 2010;116(9):1574-1584.

- Colafrancesco S, Priori R, Alessandri C, Astorri E, Perricone C, Blank M, et al. SCD163 in AOSD: a biomarker for macrophage activation related to hyperferritinemia. Immunol Res. 2014;60(23):177-183.

- Deng Y, Liu W, Liu K, Fang YY, Shang J, Zhou L, et al. Clinical characteristics of fatal and recovered cases of coronavirus disease 2019 (COVID-19) in Wuhan, China: a retrospective study. Chin Med J (Engl). 2020. https://doi.org/10.1097/CM9. 0000000000000824.

- Zhang Y, Cao W, Xiao M, Li YJ, Yang Y, Zhao J, et al. Clinical and coagulation characteristics of 7 patients with critical COVID-2019 pneumonia and acroischemia. Zhonghua Xue Ye Xue Za Zhi. 2020;41(0):E006.

- Zhang Y, Xiao M, Zhang S, Xia P, Cao W, Jiang W, et al. Coagulopathy and antiphospholipid antibodies in patients with Covid-19. N Engl J Med. 2020. Available in: https://doi. org/10.1056/NEJMc2007575.

- Hanley B, Lucas SB, Youd E, Swift B, Osborn M. Autopsy in suspected COVID-19 cases. J Clin Pathol. 2020;73:239-242. doi: 10.1136/jclinpath-2020-206522. 
- Parisi F, Paglionico A, Varriano V, Ferraccioli G, Gremese E. Refractory adult-onset still disease complicated by macrophage activation syndrome and acute myocarditis: a case report treated with high doses $(8 \mathrm{mg} / \mathrm{kg} / \mathrm{d})$ of anakinra. Medicine (Baltimore). 2017;96(24):e6656.

- Gerfaud-Valentin M, Sève P, Iwaz J, Gagnard A, Broussolle C, Durieu I, et al. Myocarditis in adult-onset still disease. Medicine (Baltimore). 2014;93(17):280-289.

- Yang J, Zheng Y, Gou X, Pu K, Chen Z, Guo Q, et al. Prevalence of comorbidities in the novel Wuhan coronavirus (COVID-19) infection: a systematic review and meta-analysis. Int $J$ Infect Dis. 2020;94:91-95. https://doi.org/10.1016/j.ijid.2020.03.017. pii: S1201-9712(20)30136-3

\section{Patrocinios: Ninguno.}

Relación de conflicto de intereses: Ninguno.

\section{Correspondencia:}

\section{Cesar Galicia García}

Av. Churubusco Núm. 601, Col. Xoco, 03339,

Alcaldía Benito Juárez, Ciudad de México, México.

Tel: 55 5623-6363,

E-mail: cesargalgar@gmail.com 